\title{
ON THE ASYMPTOTIC THEORY OF FIXED-WIDTH SEQUENTIAL CONFIDENCE INTERVALS FOR THE MEAN
}

\author{
By Y. S. Chow ${ }^{1}$ and Herbert Robbins ${ }^{2}$ \\ Purdue University and Columbia University
}

1. Introduction. Let $x_{1}, x_{2}, \cdots$ be a sequence of independent observations from some population. We want to find a confidence interval of prescribed width $2 d$ and prescribed coverage probability $\alpha$ for the unknown mean $\mu$ of the population. If the variance $\sigma^{2}$ of the population is known, and if $d$ is small compared to $\sigma^{2}$, this can be done as follows. For any $n \geqq 1$ define

$$
\bar{x}_{n}=n^{-1} \sum_{1}^{n} x_{i}, \quad I_{n}=\left[\bar{x}_{n}-d, \bar{x}_{n}+d\right],
$$

and choose $a$ to satisfy

$$
(2 \pi)^{-\frac{1}{2}} \int_{-a}^{a} e^{-u^{2} / 2} d u=\alpha .
$$

Then for a sample size $n$ determined by

$$
n=\text { smallest integer } \geqq\left(a^{2} \sigma^{2}\right) / d^{2},
$$

the interval $I_{n}$ has coverage probability

$$
P\left(\mu \varepsilon I_{n}\right)=P\left(\sqrt{n}\left|\bar{x}_{n}-\mu\right| / \sigma \leqq d \sqrt{n} / \sigma\right) .
$$

Since (1) implies that $\lim _{d \rightarrow 0}\left(d^{2} n\right) /\left(a^{2} \sigma^{2}\right)=1$, it follows from the central limit theorem that

$$
\lim _{d \rightarrow 0} P\left(\mu \varepsilon I_{n}\right)=(2 \pi)^{-\frac{1}{2}} \int_{-a}^{a} e^{-u^{2} / 2} d u=\alpha .
$$

We shall be concerned with the case in which the nature of the population, and hence $\sigma^{2}$, is unknown, so that no fixed sample size method is available. Define

$$
v_{n}=n^{-1} \sum_{1}^{n}\left(x_{i}-\bar{x}_{n}\right)^{2}+n^{-1} \quad(n \geqq 1),
$$

let $a_{1}, a_{2}, \cdots$ be any sequence of positive constants such that $\lim _{n \rightarrow \infty} a_{n}=a$, and define

$$
N=\text { smallest } k \geqq 1 \text { such that } v_{k} \leqq\left(d^{2} k\right) /{a_{k}}^{2} .
$$

The object of the present note is to prove the following

Theorem. Under the sole assumption that $0<\sigma^{2}<\infty$,

Received 5 October 1964.

${ }^{1}$ Research supported by the Office of Naval Research under Contract No. Nonr-1100(26).

2 Research supported by the Office of Naval Research under Contract No. Nonr-266(59), Project No. 042-205.

Reproduction in whole or in part is permitted for any purpose of the United States Government. 


$$
\begin{aligned}
\lim _{d \rightarrow 0}\left(d^{2} N\right) /\left(a^{2} \sigma^{2}\right) & =1 \quad \text { a.s. } \\
\lim _{d \rightarrow 0} P\left(\mu \varepsilon I_{N}\right) & =\alpha \\
\lim _{d \rightarrow 0}\left(d^{2} E N\right) /\left(a^{2} \sigma^{2}\right) & =1 .
\end{aligned}
$$

(asymptotic "consistency"),

(asymptotic "efficiency").

Remarks.

1. In case the distribution function of the $x_{i}$ is continuous, Definition (2) can be replaced by, e.g.,

$$
v_{n}=n^{-1} \sum_{1}^{n}\left(x_{i}-\bar{x}_{n}\right)^{2} .
$$

2. As will become evident from the proof, $N$ in (3) could be defined as the smallest (or the smallest odd, etc.) integer $\geqq n_{0}$ such that the indicated inequality holds, where $n_{0}$ is any fixed positive integer.

2. Proof of the theorem.

LEMma 1. Let $y_{n}(n=1,2, \cdots)$ be any sequence of random variables such that $y_{n}>0$ a.s., $\lim _{n \rightarrow \infty} y_{n}=1$ a.s., let $f(n)$ be any sequence of constants such that

$$
f(n)>0, \quad \lim _{n \rightarrow \infty} f(n)=\infty, \quad \lim _{n \rightarrow \infty} f(n) / f(n-1)=1,
$$

and for each $t>0$ define

$$
N=N(t)=\text { smallest } k \geqq 1 \text { such that } y_{k} \leqq f(k) / t .
$$

Then $N$ is well-defined and non-decreasing as a function of $t$,

$$
\lim _{t \rightarrow \infty} N=\infty \quad \text { a.s., } \quad \lim _{t \rightarrow \infty} E N=\infty,
$$

and

$$
\lim _{t \rightarrow \infty} f(N) / t=1 \text { a.s. }
$$

Proof. (9) is easily verified. To prove (10) we observe that for $N>1$, $y_{N} \leqq f(N) / t<[f(N) / f(N-1)] y_{N-1}$, whence (10) follows as $t \rightarrow \infty$.

Lemma 2. If the conditions of Lemma 1 hold and if also $E\left(\sup _{n} y_{n}\right)<\infty$, then

$$
\lim _{t \rightarrow \infty} E f(N) / t=1 \text {. }
$$

Proof. Let $z=\sup _{n} y_{n} ;$ then $E z<\infty$. Choose $m$ such that $f(n) / f(n-1) \leqq$ $2,(n>m)$. Then for $N>m$

$$
f(N) / t=[f(N) f(N-1)] /[f(N-1) t]<2 y_{N-1}<2 z .
$$

Hence for $t \geqq 1$,

$$
f(N) / t \leqq 2 z+f(1)+\cdots+f(m) .
$$

(11) follows from (10), (12), and Lebesgue's dominated convergence theorem. Proof of (4) AND (5). Set

$$
\begin{aligned}
y_{n} & =v_{n} / \sigma^{2}=\left(1 / n \sigma^{2}\right)\left(\sum_{1}^{n}\left(x_{i}-\bar{x}_{n}\right)^{2}+1\right), \\
f(n) & =\left(n a^{2}\right) / a_{n}{ }^{2}, \quad t=\left(a^{2} \sigma^{2}\right) / d^{2} ;
\end{aligned}
$$


then (3) can be written as

$$
N=N(t)=\text { smallest } k \geqq 1 \text { such that } y_{k} \leqq f(k) / t .
$$

By Lemma 1,

$$
1=\lim _{t \rightarrow \infty} f(N) / t=\lim _{d \rightarrow 0}\left(d^{2} N\right) /\left(a^{2} \sigma^{2}\right) \quad \text { a.s. }
$$

which proves (4). Now

$$
P\left(\mu \varepsilon I_{N}\right)=P\left(\left|x_{1}+\cdots+x_{N}-N \mu\right| / \sigma \sqrt{N} \leqq d \sqrt{N} / \sigma\right) .
$$

By (15), $d \sqrt{ } \bar{N} / \sigma \rightarrow a$ and $N / t \rightarrow 1$ in probability as $t \rightarrow \infty$; it follows from a result of Anscombe [1] that as $t \rightarrow \infty$,

$$
\left(x_{1}+\cdots+x_{N}-N \mu\right) / \sigma \sqrt{N} \sim N(0,1) .
$$

Hence

$$
\lim _{t \rightarrow \infty} P\left(\mu \varepsilon I_{N}\right)=(2 \pi)^{-\frac{1}{2}} \int_{-a}^{a} e^{-u^{2} / 2} d u=\alpha,
$$

which proves (5).

It remains to prove (6). This is an immediate consequence of Lemma 2 whenever the distribution of the $x_{i}$ is such that

$$
E\left\{\sup _{n}\left(n^{-1} \sum_{1}^{n}\left(x_{i}-\bar{x}_{n}\right)^{2}\right\}<\infty,\right.
$$

for then

$$
\lim _{t \rightarrow \infty}[E f(N)] / t=1,
$$

and from the fact that the function $f(n)$ defined by (14) is $n+o(n)$ it follows from (17) that

$$
1=\lim _{t \rightarrow \infty} E N / t=\lim _{d \rightarrow 0}\left(d^{2} E N\right) /\left(a^{2} \sigma^{2}\right) .
$$

For (16) to hold it would suffice for the fourth moment of the $x_{i}$ to be finite; however, we shall in the following prove that (6) holds without such a restriction. For this we need

Lemma 3. If the conditions of Lemma 1 hold, if $\lim _{n \rightarrow \infty} f(n) / n=1$, if for $N$ defined by (8),

$$
E N<\infty(\text { all } t>0), \quad \lim \sup _{t \rightarrow \infty} E\left(N y_{N}\right) / E N \leqq 1,
$$

and if there exists a sequence of constants $g(n)$ such that

$$
g(n)>0, \quad \lim _{n \rightarrow \infty} g(n)=1, \quad y_{n} \geqq g(n) y_{n-1},
$$

then

$$
\lim _{t \rightarrow \infty} E N / t=1 .
$$

Proof. For any $0<\epsilon<1$ choose $m$ so that

$$
f(n-1) \geqq(1-\epsilon) f(n)
$$




$$
\begin{aligned}
f(n-1) & \geqq(1-\epsilon) n & \text { for } n \geqq m \\
g(n) & \geqq 1-\epsilon &
\end{aligned}
$$

and $E\left(N y_{N}\right) \leqq(1+\epsilon) E N$ for $t \geqq m$. On the set $A=\{N \geqq m\}$ it follows that $\left[(1-\epsilon)^{2} / t\right] N^{2}=(1-\epsilon) N \cdot(1-\epsilon) N / t \leqq g(N) N f(N-1) / t$

$$
<g(N) N y_{N-1} \leqq N y_{N}
$$

Hence

$$
\begin{aligned}
{\left[(1-\epsilon)^{2} / t\right]\left(\int_{A} N\right)^{2} } & \leqq\left[(1-\epsilon)^{2} / t\right] \int_{A} N^{2} \leqq \int_{A} N y_{N} \leqq E\left(N y_{N}\right) \\
{\left[(1-\epsilon)^{2} / t\right] \int_{A} N } & \leqq E\left(N y_{N}\right) / \int_{A} N \\
{\left[(1-\epsilon)^{2} / t\right](E N-m) } & \leqq E\left(N y_{N}\right) /(E N-m)
\end{aligned}
$$

From (9) and (18) it follows that

$$
(1-\epsilon)^{2} \lim \sup _{t \rightarrow \infty} E N / t \leqq \lim \sup _{t \rightarrow \infty} E\left(N y_{N}\right) /(E N) \leqq 1,
$$

so that

$$
\lim \sup _{t \rightarrow \infty} E N / t \leqq 1 .
$$

Now let $y_{n}{ }^{\prime}=\min \left(1, y_{n}\right)$. Then

$$
0<y_{n}^{\prime} \leqq 1, \quad y_{n}^{\prime} \leqq y_{n}, \quad \lim _{n \rightarrow \infty} y_{n}{ }^{\prime}=1 \text { a.s. }
$$

Define

$$
N^{\prime}=N^{\prime}(t)=\text { smallest } k \geqq 1 \text { such that } y_{k}{ }^{\prime} \leqq f(k) / t .
$$

From Lemma 2, since $\sup _{n}\left(y_{n}{ }^{\prime}\right) \leqq 1$,

$$
1=\lim _{t \rightarrow \infty}[E f(N)] / t=\lim _{t \rightarrow \infty}\left(E N^{\prime}\right) / t .
$$

But since $y_{n}{ }^{\prime} \leqq y_{n}, N^{\prime} \leqq N$, and hence $E N^{\prime} \leqq E N$. Thus

$$
\lim \inf _{t \rightarrow \infty}(E N) / t \geqq \lim \inf _{t \rightarrow \infty}\left(E N^{\prime}\right) / t=1,
$$

which, with (20), proves (19).

Proof of (6). Fix $t>0$, choose $m$ such that $f(n) / t \geqq 1(n \geqq m)$, choose $\delta>0$ such that $(n-1) f(n-1) \geqq \delta n^{2}(n \geqq 2)$, and define for any $r \geqq m$, $M=\min (N, r)$. By Wald's theorem for cumulative sums,

$$
E\left(\sum_{1}^{M}\left(x_{i}-\mu\right)^{2}\right)=E \dot{M} \cdot E\left(x_{i}-\mu\right)^{2}=E M \cdot \sigma^{2} .
$$

Hence by (13),

$$
\begin{aligned}
E\left(M y_{M}\right) & =\left(1 / \sigma^{2}\right) E\left(\sum_{1}^{M}\left(x_{\imath}-\bar{x}_{M}\right)^{2}+1\right) \\
\leqq & \left(1 / \sigma^{2}\right) E\left(\sum_{1}^{M}\left(x_{i}-\mu\right)^{2}+1\right)=E M+\left(1 / \sigma^{2}\right) .
\end{aligned}
$$

Put $g(n)=(n-1) / n,(n \geqq 2) ;$ then 
$y_{n} \geqq\left(1 / n \sigma^{2}\right) \sum_{1}^{n-1}\left(x_{i}-\bar{x}_{n-1}\right)^{2}+\left(1 / n \sigma^{2}\right)=[(n-1) / n] y_{n-1}=g(n) y_{n-1}$.

Hence

$$
\begin{array}{r}
E\left(M y_{M}\right) \geqq \int_{\{N>r\}} r y_{r}+\int_{\{N \leqq r\}} N y_{N} \geqq[r f(r) / t] P(N>r)+\int_{\{2 \leqq N \leqq r\}} N y_{N} \\
\geqq r P(N>r)+\int_{\{2 \leqq N \leqq r\}}[N g(N) f(N-1)] / t \\
\geqq r P(N>r)+(\delta / t) \int_{\{2 \leqq N \leqq r\}} N^{2} .
\end{array}
$$

Hence by. (21),

$$
\int_{\{N \leqq r\}} N \geqq(\delta / t) \int_{\{2 \leqq N \leqq r\}} N^{2}-\left(1 / \sigma^{2}\right) \geqq(\delta / t)\left(\int_{\{2 \leqq N \leqq r\}} N\right)^{2}-\left(1 / \sigma^{2}\right),
$$

and letting $r \rightarrow \infty$ it follows that

$$
E N=\lim _{r \rightarrow \infty} \int_{\{N \leqq r\}} N<\infty,
$$

which is the first part of (18). Again by Wald's theorem,

$$
E\left(N y_{N}\right) \leqq E N+\left(1 / \sigma^{2}\right)
$$

so by (9),

$$
\lim \sup _{t \rightarrow \infty}\left[E\left(N y_{N}\right)\right] /(E N) \leqq 1,
$$

which is the second part of (18). All the conditions of Lemma 3 therefore hold, and hence

$$
1=\lim _{t \rightarrow \infty} E N / t=\lim _{d \rightarrow 0}\left(d^{2} E N\right) /\left(a^{2} \sigma^{2}\right),
$$

which is (6). This completes the proof of the theorem of Section 1. As to Remark 1 following the theorem, it is clear that the only purpose of the term $n^{-1}$ in (2) is to ensure that $y_{n}=v_{n} / \sigma^{2}>0$ a.s., this fact having been used in the proof of Lemma 1 to guarantee that $N \rightarrow \infty$ a.s. as $t \rightarrow \infty$. If the distribution function of the $x_{i}$ is continuous the definition ( 7 ) is equally good, the only change being that the term $1 / \sigma^{2}$ in the proof of (6) disappears.

The method used in this note is a modification of that used in [3] to prove the elementary renewal theorem. The theorem in this note has been proved when the $x_{i}$ are $N\left(\mu, \sigma^{2}\right)$ by Stein [6], Anscombe [1], [2], and Gleser, Robbins, and Starr [4]. Some numerical computations for a slightly modified procedure have been made by Ray [5] who, apparently misled by having considered too few values of $d$, doubts the validity of (5) in his case. Extensive numerical computations in the $N\left(\mu, \sigma^{2}\right)$ case have been made by Starr and will soon be available. They indicate, for example, that for $\alpha=.95$ the lower bound for all $d>0$ of $P\left(\bar{x}_{N}-d \leqq \mu \leqq \bar{x}_{N}+d\right)$, where $N$ is the smallest odd integer $k \geqq 3$ such that

$$
(k-1)^{-1} \sum_{1}^{k}\left(x_{i}-\bar{x}_{k}\right)^{2} \leqq\left(d^{2} k\right) / a_{k}^{2},
$$

is about .929 if the values $a_{k}$ are taken from the $t$-distribution with $(k-1)$ degrees of freedom. 


\section{REFERENCES}

[1] Anscombe, F. J. (1952). Large sample theory of sequential estimation. Proc. Cambridge Philos. Soc. 48 600-607.

[2] Anscombe, F. J. (1953). Sequential estimation. J. Roy. Stat. Soc. Ser. B 15 1-21.

[3] Dоов, J. L. (1948). Renewal theory from the point of view of the theory of probability. Trans. Amer. Math. Soc. 63 422-438.

[4] Gleser, L. J., Robbins, H., and Starr, N. (1964). Some asymptotic properties of fixedwidth sequential confidence intervals for the mean of a normal population with unknown variance. Report on National Science Foundation Grant NSF-GP2074, Department of Mathematical Statistics, Columbia University.

[5] RAY, W. D. (1957). Sequential confidence intervals for the mean of a normal distribution with unknown variance. J. Roy. Stat. Soc. Ser. B 19 133-143.

[6] Stein, C. (1949). Some problems in sequential estimation. Econometrica 17 77-78. 